\title{
Development of a PC-Based Software Receiver for the Reception of Beidou Navigation Satellite Signals
}

\author{
Jyh-Ching Juang, Chiu-Teng Tsai and Yu-Hsuan Chen \\ (Department of Electrical Engineering, National Cheng Kung University, Taiwan) \\ (E-mail: juang@mail.ncku.edu.tw)
}

\begin{abstract}
Beidou is the Global Navigation Satellite System (GNSS) being developed in China, with the aim to provide a global navigation service that is similar to the Global Positioning System (GPS) and Galileo navigation systems. In this paper, it is demonstrated that through the flexibility and re-configurability of a PC-based software receiver in which the baseband operations are realized in terms of software, it is possible to acquire, track, and demodulate Beidou satellite signals even when only a limited amount of information is known. Further, with the Beidou interface control document now available, the proposed PC-based software receiver can be easily adapted to perform navigation functions. This research lays the foundation for the further development of navigation receivers and exploration of multiGNSS applications.
\end{abstract}

KEY WORDS

1. Global Navigation Satellite System (GNSS). 2. Beidou. 3. Software receiver.

Submitted: 16 October 2012. Accepted: 2 May 2013. First published online: 12 June 2013.

1. INTRODUCTION. Global Navigation Satellite Systems (GNSS) have evolved rapidly in recent years as several newly developed navigation satellites including the modernized GPS IIF, Galileo IOV (In-Orbit Validation), Beidou and GLONASS-K satellites have been deployed. Together, they will affect many commercial, military and social location-relevant activities. As of today, the GPS Block IIF satellites are starting to broadcast an operational L5 signal, which is the third civilian GPS signal for positioning, navigation, and timing (PNT). Four Galileo IOV satellites have been launched to validate the Galileo design and pave the way for the full deployment of the Galileo constellation. To be compatible with other navigation satellite systems, the GLONASS-K satellite is starting to test civilian navigation in Code Division Multiple Access (CDMA) signals, in addition to the conventional Frequency Division Multiple Access (FDMA) signals. Of note, in late 2011, GLONASS achieved a constellation of 24 operational spacecraft with the ability to provide full coverage of the Earth's surface. In addition, several regional satellitebased navigation/augmentation systems such as the Quasi-Zenith Satellite System 
(QZSS) are being realized. Thus, GNSS have become a multi-constellation, multisignal system of systems.

The Beidou satellite navigation system (BDS) is being developed in China and aims to provide a global navigation service that is similar to GPS and GLONASS navigation systems. According to the China National Space Administration (CNSA), from 2012, the Beidou system is providing a navigation service in the Asia-Pacific region and, by 2020, Beidou will eventually become a global navigation system in which the space segment will consist of five geostationary (GEO) satellites, three inclined geostationary (IGSO) satellites, and 27 medium earth orbiting (MEO) satellites (Beidou Navigation Satellite System, 2012). Beidou navigation satellites will broadcast CDMA navigation signals in multiple bands. The signals are expected to be compatible with other GNSS signals; it is thus interesting to develop receiver techniques to acquire and track Beidou signals and to perform positioning by using Beidou signals so as to assess the signal quality and seek the synergistic use of multi-GNSS signals. In this paper, a personal computer (PC)-based GNSS software receiver is developed to receive Beidou signals. The software receiver approach is adopted as there are no Beidou receivers commercially available at the time of development and the detailed signal definition of Beidou is evolving. The contributions of the paper are as follows:

- Although the significance of software-defined radio (SDR) has been advocated in wireless communication and cognitive radio, the use of SDR techniques in the reception of GNSS signals requires some unique design features to fulfil the requirements of real-time operation and tracking with a relatively weak signal power level. This paper presents a software-based approach to realize GNSS signal tracking function in real-time.

- Two versions of the Beidou ICD (interface control document) were released at the end of 2011 and 2012, respectively. The test version (China Satellite Navigation Office, 2011) contains descriptions of the B1I signal specifications and ranging code while the ICD (China Satellite Navigation Office, 2012) additionally provides information on the navigation message, including parameters of the satellite almanacs and ephemerides of the open service B1I signals. The paper demonstrates the potential and flexibility of SDR in keeping pace with the release of the ICD.

- Since Taiwan is geographically located in the footprint of Beidou satellites, the application of the PC-based software receiver can thus provide an independent assessment of the Chinese Beidou navigation satellite signals.

- A benefit of the SDR is that the receiver functions can be easily and progressively modified to account for unique signal features that are not known a priori. Such a characteristic is exploited in the paper to track Beidou signals and demodulate data messages. Based on the received and demodulated data, the paper presents some observations on the Beidou signals, made when only the test version was available publically. The decoding and positioning functions can be established once the formal ICD is available.

The paper discusses some related works on GNSS software receivers and Beidou navigation satellite system in Section 2. The design and implementation of the PC-based software receiver are presented in Section 3. Section 4 provides the 
acquisition, tracking, and positioning results by using the software receiver in processing Beidou satellite signals. Finally, Section 5 gives the conclusion.

2. RELATED WORK. A GNSS receiver typically comprises an antenna, radio frequency (RF) processing unit, baseband processing unit, and navigation processing unit. The RF processing unit performs the tasks of amplification, filtering, down-conversion and analogue-to-digital conversion to produce data samples in the intermediate frequency (IF) band. The baseband processing unit is responsible for the acquisition, tracking and data demodulation tasks so that raw GNSS ranging measurements can be obtained and navigation data can be decoded. The navigation processing unit, in turn, computes position, velocity and time. Existing GNSS receivers typically rely on a baseband chip to carry out the baseband processing functions and an embedded processor to realize the navigation processing tasks. In a GNSS software receiver, the baseband and navigation processing functions are performed at the host computer. This can be a field programmable gate array (FPGA), digital signal processor (DSP), PC, graphical processing unit (GPU) or a combination thereof. The use of software receivers for GNSS signal reception is noted for their flexibility, re-configurability, and diversity.

For GPS signals reception and processing, several software receivers have been reported. Tsui (2000) and Borre et al. (2007) discussed suitable GNSS signal processing algorithms for software receivers. Suitable algorithms for GNSS signal acquisition can be found in Sagiraju et al. (2008) and Juang and Chen (2010). In a software receiver, the correlation operation is the most demanding task, and a bit-wise parallel method was proposed by Ledvina et al (2004a) to improve the efficiency of this. In addition, a unique implementation technique to realize the correlation operation was discussed by Heckler and Garrison (2006). To account for the unique arrangement of phase tables in software receivers, a frequency/phase tracking method was investigated by Juang and Chen (2009a). Early attempts to produce real-time GPS software receivers were made by Akos (1997) and Ledvina et al. (2004b). More recently, GPS software that is capable of processing both L1 and L5 signals in real time has been reported by Chen et al. (2011). In real-time software receivers, the data intermittency issue cannot be neglected and a compensation scheme is proposed by Juang and Chen (2009b) to address this issue.

This paper attempts to develop and apply software receiver techniques to receive Beidou satellite signals. The Beidou constellation employs three different orbits, GEO, IGSO and MEO, to balance the need of regional and global coverage. Each Beidou satellite plans to broadcast signals in the B1, B1_2, B2, and B3 frequency bands. In the test-version ICD of BDS (China Satellite Navigation Office, 2011), the modulation and spreading code of the B1 I channel signals are described. The B1 I channel spreading code is a truncated Gold code of length 2046 chips with a chipping rate of 2.046 Mcps (chips per sec). The constituting polynomials are $1+x+x^{7}+x^{8}+x^{9}+x^{10}+x^{11}$ and $1+x+x^{2}+x^{3}+x^{4}+x^{5}+x^{8}+x^{9}+x^{11}$, respectively. The B1 frequency is at $1561.098 \mathrm{MHz}$, which is close to GPS L1 frequency. Table 1 compares the key parameters of the GPS and Beidou open service signals. Figure 1 depicts the GNSS signal spectrum in the high L band from $1.5 \mathrm{GHz}$ to $1.7 \mathrm{GHz}$. The Beidou signals are close in frequency to GPS/Galileo signals. It is expected that all the signal components can be explored in future GNSS receivers. Previously, some signal 
Table 1. Comparison of GPS and BDS open service signals.

\begin{tabular}{|c|c|c|c|}
\hline \multirow[b]{2}{*}{ System } & \multirow[b]{2}{*}{ GPS } & \multicolumn{2}{|l|}{ BDS } \\
\hline & & IGSO/MEO & GEO \\
\hline Center frequency & $1575 \cdot 42 \mathrm{MHz}$ & \multicolumn{2}{|c|}{$1561.098 \mathrm{MHz}$} \\
\hline Modulation & BPSK & \multicolumn{2}{|c|}{ QPSK } \\
\hline Spreading code & C/A code; 10 -stage Gold code & \multicolumn{2}{|c|}{ Truncated 11-stage Gold code } \\
\hline Code length & 1023 & \multicolumn{2}{|c|}{2046} \\
\hline Chipping rate & 1.023 Mcps & \multicolumn{2}{|l|}{ 2.046 Mcps } \\
\hline Code period & $1 \mathrm{msec}$ & \multicolumn{2}{|l|}{$1 \mathrm{msec}$} \\
\hline Coding scheme & Parity check & \multicolumn{2}{|c|}{$\mathrm{BCH}(15,11,1)+$ interleave } \\
\hline Secondary code & $\mathrm{n} / \mathrm{a}$ & Neumann-Hoffman code & $\mathrm{n} / \mathrm{a}$ \\
\hline Data rate & $50 \mathrm{bps}$ & $50 \mathrm{bps}$ & $500 \mathrm{bps}$ \\
\hline Coordinate system & WGS-84 & \multicolumn{2}{|l|}{ CGCS2000 } \\
\hline Time system & GPST & BDT & \\
\hline
\end{tabular}

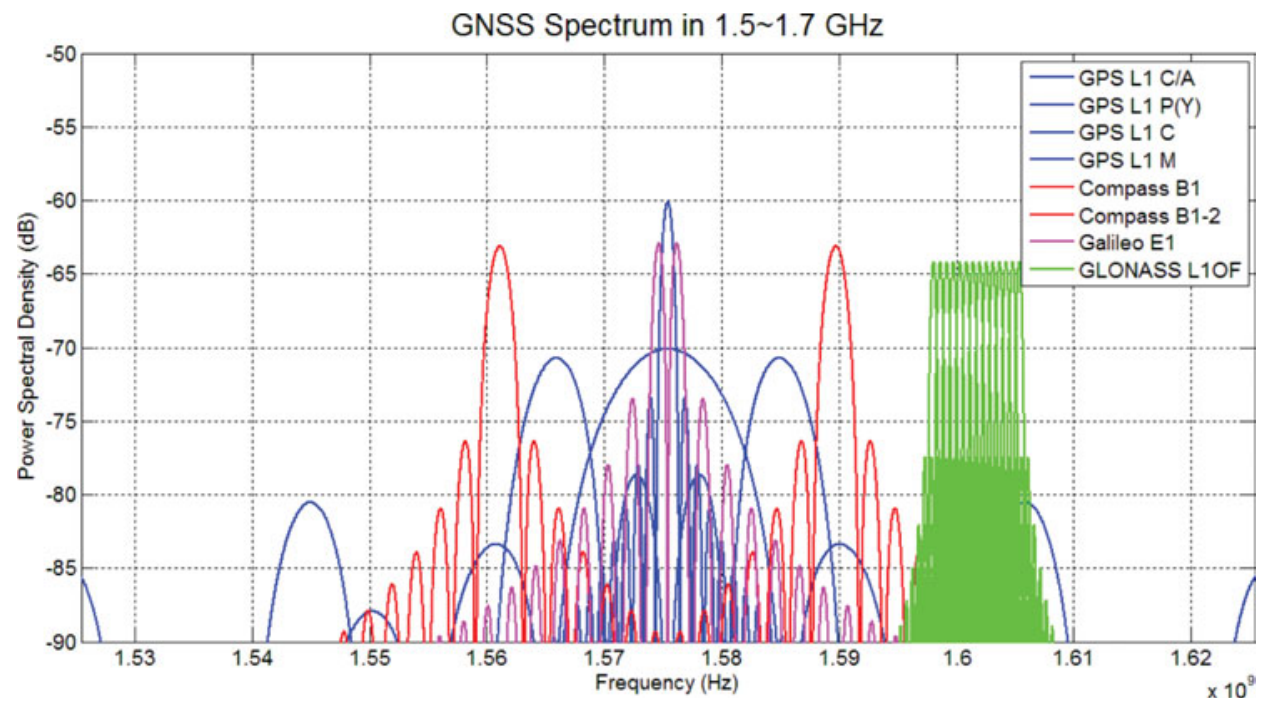

Figure 1. The spectrum of current GNSS signals in $1.5 \sim 1.7 \mathrm{GHz}$.

analysis results on the Beidou MEO M1 satellite have been reported by Gao et al. (2009) and Hauschild et al. (2012). The aim of this work is to develop key enabling techniques to receive Beidou broadcast signals for the eventual integration of all GNSS signal components. Moreover, all MEO, GEO, and IGSO satellite signals are tracked in this paper. In the ICD of BDS (China Satellite Navigation Office, 2012), the navigation messages are described. Another goal of the paper is to perform the positioning task and assess performance.

3. SOFTWARE RECEIVER FOR THE RECEPTION OF BEIDOU SIGNALS. In this section, the design and implementation of a PC-based software receiver for the reception of Beidou signals will be described. 


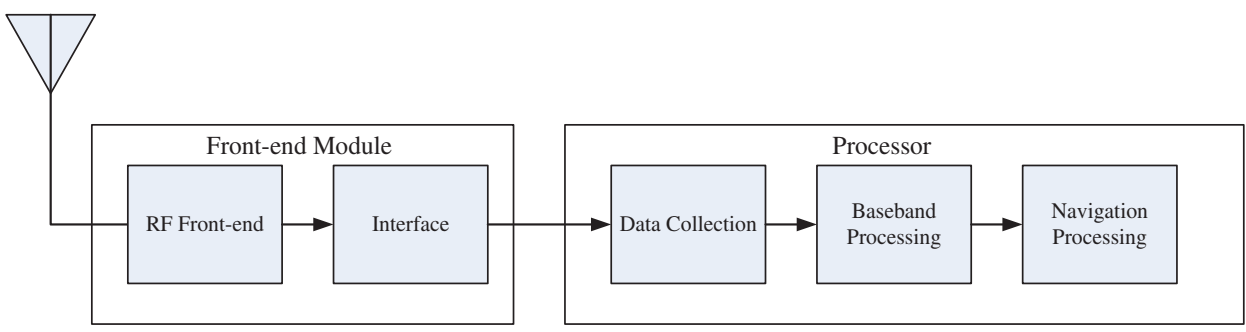

Figure 2. Schematic of a GNSS software receiver.

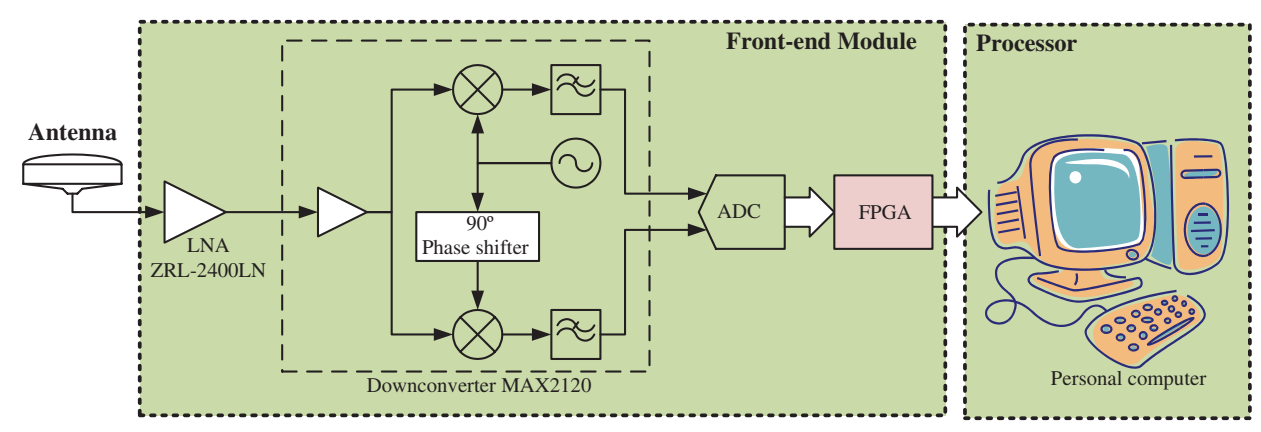

Figure 3. The architecture of a Beidou signal receiver.

3.1. GNSS Software Receiver. As previously mentioned, a GNSS software receiver consists of three elements, RF processing unit, baseband processing unit, and navigation processing unit. The RF processing unit or RF front-end module is responsible for signal amplification, noise filtering, down-conversion, automatic gain control, and analogue-to-digital conversion. The front-end module renders digital IF data at a rate of several $\mathrm{MHz}$ even though the incoming GNSS signal is in the L band. The digital IF data are then processed by a baseband processing unit whose roles include correlation, signal acquisition, code and carrier tracking and data demodulation. The demodulated data and resulting pseudorange measurements are then processed by a navigation processing unit to yield position, velocity, timing, and other relevant information. The software receiver differs from a conventional receiver in the sense that the functions of the baseband chip, including correlation/tracking and navigation tasks, are delivered by software, leading to a more flexible design with potential savings in cost and power. In practice, the software is executed in a processor that can either be an FPGA, DSP, PC, GPU, etc. Figure 2 depicts the schematic of a typical GNSS software receiver.

For the reception of Beidou signals, the PC platform is adopted as it is supported with a comprehensive software development environment and the associated multicore architecture can be used to achieve the desired real-time performance. The hardware implementation of the PC-based Beidou software receiver is depicted in Figure 3. A multi-band GNSS antenna with the operating frequency range covering the Beidou B1 band is employed. The received signal is amplified by a low-noise amplifier (LNA) before it is sent to the down-converter. The system utilizes a broadband in-phase/quadrature (I/Q) down-converter to convert the RF signals into 


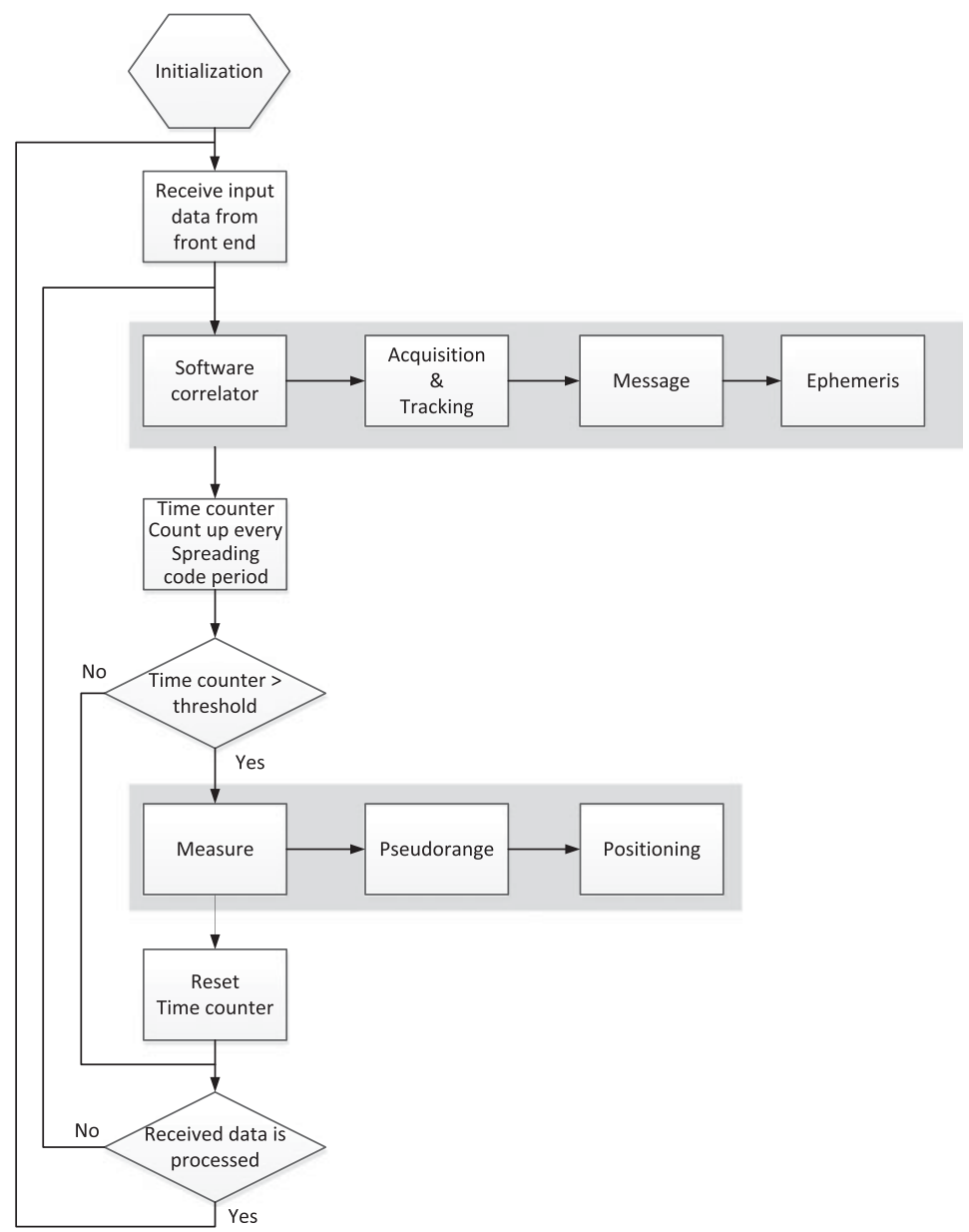

Figure 4. Flow chart of the GNSS software receiver.

IF band. For the reception of the $1561.098 \mathrm{MHz}$ Beidou B1 signal, the LO (local oscillator) frequency is set at $1561 \mathrm{MHz}$ and, as a result, the IF is $0.098 \mathrm{MHz}$. The IF I/Q signals are then digitized by an analogue-to-digital converter with a sampling frequency of 4 Msps (samples per sec). An FPGA is then used to buffer and pack the digitized signal samples in the desired format for the access of the PC. The digital data are then processed by the software in the $\mathrm{PC}$ where all the baseband and navigation processing functions are performed.

The receiver functions, including acquisition, tracking, demodulation, decoding and navigation are implemented in software, mostly developed with Visual Studio $\mathrm{C}++$ in Microsoft Windows 7. The flow chart of the software receiver is depicted in Figure 4. The software starts with the initialization routine in which filter coefficients, channel assignments, variables pertaining to the setting of tracking channel, initial estimates of position and time, almanac, and ephemeris are initialized. After the initialization, the software enters an indefinite loop in which a block of data from the front end is obtained and processed. For Beidou signal processing, the size of the block corresponds to the period of the spreading code, which is $1 \mathrm{msec}$. The data are then 

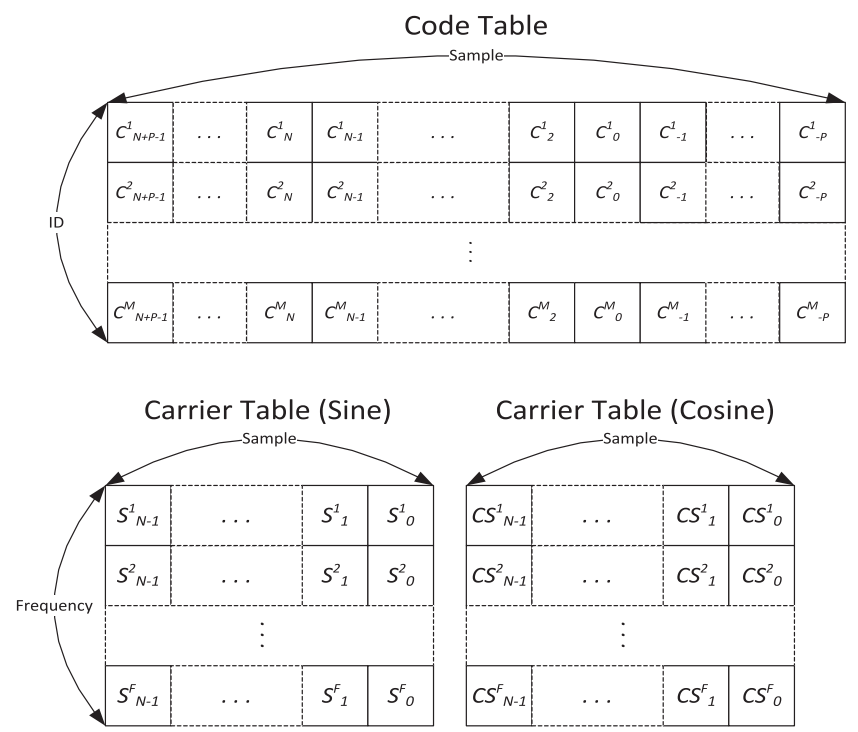

Figure 5. Structures of code table and carrier table.
C: Code

S: Sine

CS: Cosine

I: IF Data I

Q: IF Data Q

$\mathrm{N}$ : \# of samples in $1 \mathrm{msec}$

$\mathrm{M}$ : \# of ID

$P$ : \# of samples in

E-P spacing

F: \# of Frequency

correlated with local replicas in the software correlator. The correlation results are fed to the tracking procedure so that the data can be demodulated. Consequently, the message, measure and positioning functions are performed. It is noted, however, that the main challenge in the software receiver is the signal de-spreading, acquisition, and tracking tasks, which need to be realized at a high computational rate with a large amount of data samples. By comparison, the message, measure, and positioning functions are less demanding.

3.2. Software Correlator. The most demanding task in a GNSS software receiver is software correlation, which needs to be executed at a high rate. In order to implement the correlation function in software, tables of code and carrier are provided and stored in memory before execution. For Beidou B1 signal processing, the tables are made $1 \mathrm{msec}$ long and its number of phases is 4000 which corresponds to the number of samples in $1 \mathrm{msec}$. A code table is made for each satellite. The carrier table is made to span $\pm 10 \mathrm{kHz}$ Doppler frequency with a spacing of $10 \mathrm{~Hz}$. To reduce the size of the carrier table, each replica of frequency component starts from zero phase. Figure 5 shows the structures of code table and carrier table where the size of one sample is 1 bit for coarse tracking or several bits for fine tracking.

To achieve real-time operation, the software correlator is often implemented in a parallel approach. The bit-wise parallel algorithm represents each sample of signal by bits and performs bit-wise operations to conduct the correlation operation more efficiently (Ledvina et al. 2004a). In the PC environment, Single Instruction Multiple Data (SIMD) instruction sets can be employed to perform the same operations on multiple data (Heckler and Garrison, 2006). For current x86 processors, one of the SIMD instruction sets is Streaming SIMD Extension (SSE) instructions, which can allocate multiple samples in one 128-bit-wide XMM register and perform parallel operations in one instruction cycle. In the software receiver, the bit-wise parallel algorithm is generalized to account for the need for coarse and fine tracking and implemented using the SIMD instructions. As a result, 128 samples can be processed 


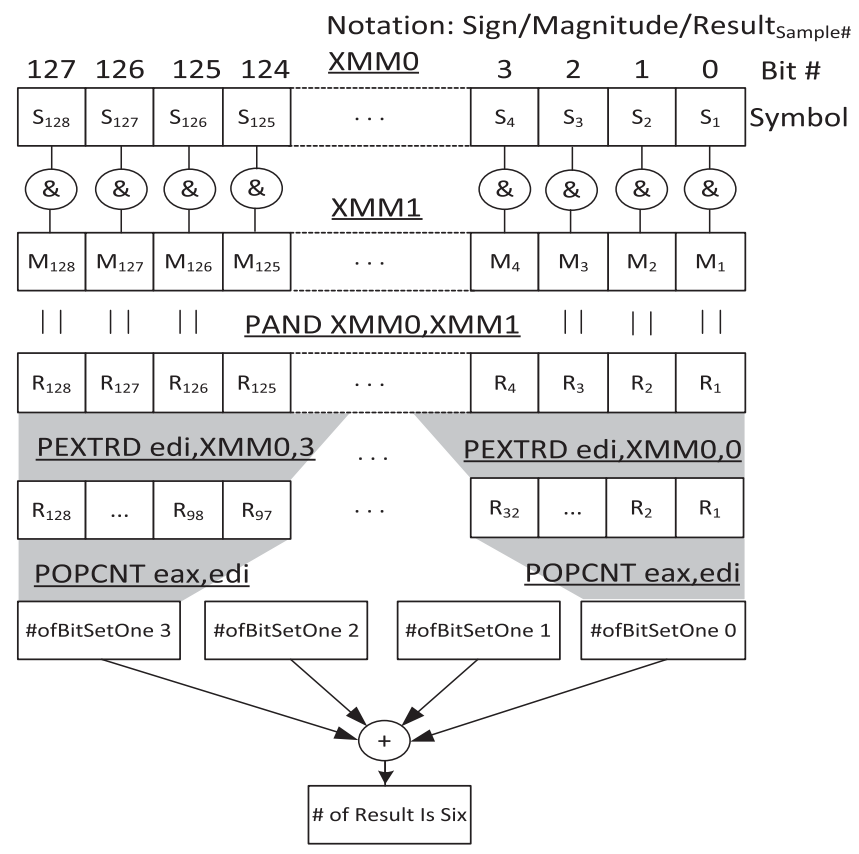

Figure 6. Example of counting the number of correlation results via SSE instructions.

at a time using the 128-bit XMM registers. After the bit-wise operation, the results are stored within the bits of the register and an accumulation is required. To this end, the POPCNT instruction is used to count the number of bits set to one for a 32-bit register in a 32-bit operating system. An example of counting the number of correlation results using SSE instructions is shown in Figure 6.

3.3. Acquisition/Tracking Procedure. In the software receiver, the carrier tracking loop is different from the traditional one (Holmes, 2007) because the carrier table is made for limited numbers of frequencies and has zero phase in the beginning of the table. The incoming data is mixed with the local carrier phase replica every msec to generate I and Q correlation outputs. A rotation operation is then employed to account for zero-phase tabular data. The amount of rotation depends on the frequency as well as integration interval. The rotated I and Q components are then passed to a phase discriminator and the phase error as well as the frequency error is obtained. The phase error is then filtered and used to adjust the frequency of the local oscillator in the look-up table. The entire acquisition/tracking procedure is controlled by a state machine with four states: acquire, confirm, pull-in, and lock as shown in Figure 7. The acquire state performs a search in the dimensions of Doppler frequency and code phase to detect the presence of Beidou signals. As long as the magnitude of any correlators is greater than a pre-determined threshold, the operation then enters the confirm state. In the confirm state, an $\mathrm{M}$ of $\mathrm{N}$ detector is used to confirm the presence of the signal. Once the signal is detected, the operation then enters the pull-in state. In the pull-in state, a frequency locked loop (FLL) plus phase locked loop (PLL) carrier tracking loop (Juang and Chen, 2009a) and a delay locked loop (DLL) code tracking loop are used to pull in the carrier frequency and code phase, respectively. After going through the pull-in process for a specific time period, the software will 


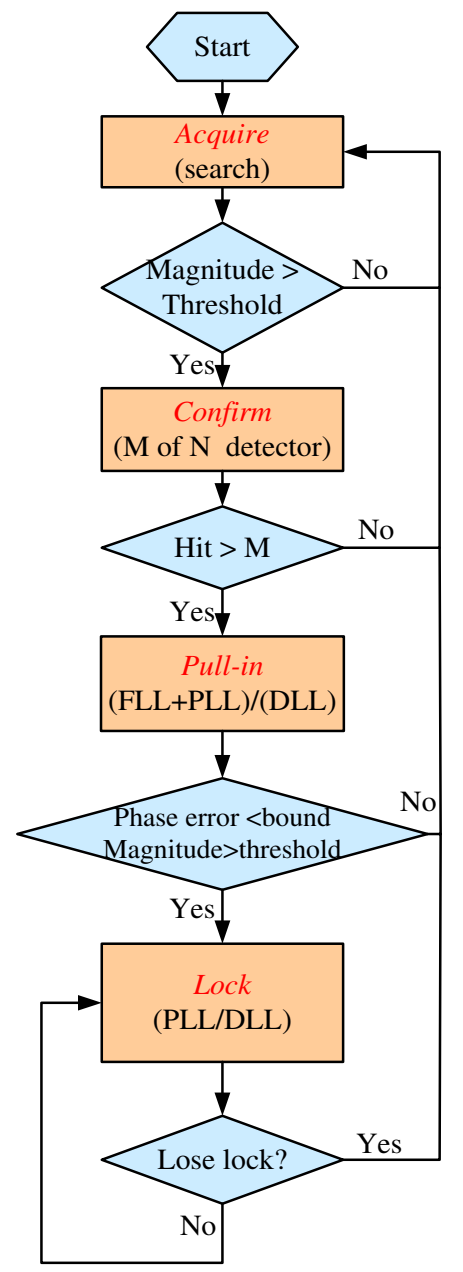

Figure 7. Tracking procedure state machine.

check whether the variance of the phase error is under the accepted level and the magnitude of the correlation output of the prompt correlator is greater than the threshold. If affirmative, the tracking operation enters the lock state. In the lock state, both the PLL carrier locked loop and the DLL have a narrower noise bandwidth to reduce the tracking error. The software will periodically check whether the signal is lost. If it is, the tracking procedure returns to the acquire state.

3.4. Navigation Procedure. Once the signals are successfully locked, navigation data can then be decoded for the determination of the user's position, velocity, and time (PVT) as depicted in Figure 4. Typically, the navigation procedure of a software receiver is similar to that of a traditional receiver and the tasks to be performed are subframe alignment, data decoding, error correction, navigation message extraction, pseudorange measurement, satellite position and time computation, and PVT determination. In performing the BDS navigation tasks, it is noted that the arrangements of the IGSO/MEO data and the GEO data are not the same. Thus, two different routines are developed for the extraction of navigation messages. 
Table 2. Orbits and status of Bediou satellites (as of 2011).

\begin{tabular}{llll}
\hline Satellite & Launch date & \multicolumn{1}{c}{ Orbit } & \multicolumn{1}{c}{ status } \\
\hline M1 & $2007 / 04 / 14$ & MEO & Normal (Testing only) \\
G2 & $2009 / 04 / 15$ & GEO & Unusable (orbit drifting) \\
G1 & $2010 / 01 / 17$ & GEO $\left(140^{\circ} \mathrm{E}\right)$ & Normal \\
G3 & $2010 / 06 / 02$ & GEO $\left(84^{\circ} \mathrm{E}\right)$ & Normal \\
I1 & $2010 / 08 / 01$ & IGSO & Normal \\
G4 & $2010 / 11 / 01$ & GEO $\left(160^{\circ} \mathrm{E}\right)$ & Normal \\
I2 & $2010 / 12 / 18$ & IGSO & Normal \\
I3 & $2011 / 04 / 10$ & IGSO & Normal \\
I4 & $2011 / 07 / 27$ & IGSO & Normal \\
I5 & $2011 / 12 / 01$ & IGSO & Normal \\
\hline
\end{tabular}

In either case, features including preamble detection, $\mathrm{BCH}(15,11,1)$ decoding, data interleaving and ephemeris extraction are implemented. In particular, the seconds-ofweek messages are used, together with the code tracking result, to facilitate the determination of the code pseudoranges. Once the ephemeris parameters are obtained, the position and clock error correction of the satellite can be computed. The ephemeris algorithm of the IGSO/MEO satellites and that of the GEO satellites are slightly different as described in the Beidou ICD (China Satellite Navigation Office, 2012). Upon the availability of the pseudorange measurements and satellite ephemeris, the position and clock offset of the user can be determined using the linearization and weighted Least Squares method. With these features in place, the software receiver is capable of performing the Beidou navigation function.

4. RECEPTION OF BEIDOU SIGNALS. In this section, the results of using a PC-based software receiver in the acquisition, tracking, and navigation of Beidou signals are presented. Two signal reception experimental campaigns were conducted in the campus of National Cheng Kung University, Taiwan, one at local time 20:39, January 11, 2012 and the other at 12:55, January 11, 2013. Note that at the first experiment, only the test version ICD was available, while for the second experiment, the formal ICD had been released. In the following, the acquisition, tracking, and demodulation results of the first campaign and the navigation result of the second campaign are reported.

4.1. Experiment 1. Table 2 depicts the list of Beidou satellites in orbit at the time of this experiment. Three categories of Beidou satellites at MEO, GEO, and IGSO, respectively, are employed to achieve a balanced regional and global coverage. The sky plot is illustrated in Figure 8, which clearly indicates that all the normal Beidou satellites can be observed. The PC-based software receiver was then employed to acquire and track these Beidou satellite signals. This experiment focused on acquisition and tracking.

4.1.1. Acquisition. For the Beidou B1 I channel signal, the chipping rate is $2.046 \mathrm{Mcps}$; the sampling rate of the software receiver is selected to be 4.000 Msps. The objective of acquisition is to determine the visibilities of satellites and estimates of Doppler frequency and code phase. The acquisition is implemented using a parallel code-phase search scheme through the Fast Fourier transform (FFT) -based 
Skyplot of Beidou Satellites

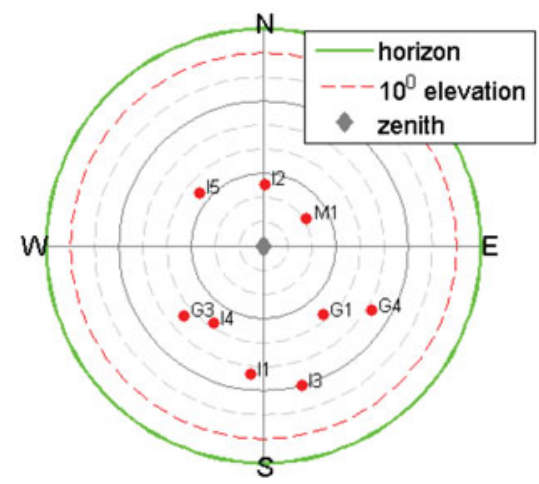

Figure 8. Sky plot of Beidou navigation satellites at the time of experiment.

processing (Tsui, 2000). More precisely, the ranging code is generated, resampled, transformed and conjugated. The incoming digital IF samples are mixed with the local carrier replica at the chosen Doppler frequency before the samples are transformed. The transformed signal samples and codes are multiplied sample by sample and, afterwards, undergo an inverse transformation. The magnitudes of the inversetransformed variables are then examined for the detection of the presence of the signal. The FFT-based processing is known to able to speed up the acquisition process. In the acquisition process, the resolution of Doppler frequency is $125 \mathrm{~Hz}$. The acquisition result of the Beidou satellite B1 I channel signals is depicted in Figure 9. As there are nine satellites in the sky, nine subplots are presented in the figure. In each 3-D subplot, the magnitude of the correlation output as a function of the Doppler frequency (x-axis) and code phase (y-axis) is plotted. The Doppler frequency is in the unit of $\mathrm{KHz}$ and the code phase is in the unit of chip. In each subplot, a peak can be clearly observed, implying that the corresponding satellite signal is successfully acquired. The code phase and Doppler frequency associated with this satellite signal can then be read for the preparation of signal tracking. Table 3 depicts the code phase, Doppler frequency and the post-correlation carrier-to-noise $(\mathrm{C} / \mathrm{N})$ of the acquired signals, together with the elevation angle of the satellite. The code phase is in the unit of sample and 4000 samples correspond to 2046 chips. By comparing the $\mathrm{C} / \mathrm{N}$ in Table 3 against the sky plot in Figure 8, it is clear that the low-elevation satellites are subject to a more severe path loss and, as a result, a lower $\mathrm{C} / \mathrm{N}$.

4.1.2. Tracking. With the availability of coarse estimates of the Doppler frequency and code phase, we then attempted to track each signal. Figure 10 depicts the tracking results of G1 satellite signals. In the figure, the in-phase prompt correlator output, code error, phase error, and Doppler frequency estimate are presented. After the transient period, the Doppler frequency estimate converges, both the phase error and code error are stabilized, and the signal is locked. When locked, the sign of the inphase prompt signal is related to the data bit and its magnitude is related to $\mathrm{C} / \mathrm{N}$. As the B1 I channel signal is BPSK modulated, the signal constellation diagram can be used to illustrate the tracking performance. Figure 11 depicts the constellation diagrams of the signals from nine different satellites. It is clear from the figure that all the signals can be demodulated and decoded. In Figure 11, the quadrature prompt 
Table 3. Acquisition results.

\begin{tabular}{lccrc}
\hline Satellite & Code phase (sample) & Doppler frequency $(\mathrm{Hz})$ & $\mathrm{C} / \mathrm{N}(\mathrm{dB})$ & Elevation $(\mathrm{deg})$ \\
\hline G1 & 1697 & -2500 & $12 \cdot 641$ & $52 \cdot 2893$ \\
G3 & 3688 & -2625 & $12 \cdot 016$ & $46 \cdot 0485$ \\
G4 & 1874 & -2750 & $9 \cdot 269$ & $36 \cdot 6895$ \\
I1 & 3078 & -4125 & $7 \cdot 026$ & $38 \cdot 0912$ \\
I2 & 3307 & -2750 & $10 \cdot 159$ & $64 \cdot 4518$ \\
I3 & 1787 & -1000 & $7 \cdot 194$ & $29 \cdot 9907$ \\
I4 & 3374 & -3875 & $10 \cdot 067$ & $51 \cdot 8699$ \\
I5 & 1712 & -3000 & $8 \cdot 698$ & $55 \cdot 5749$ \\
M1 & 1037 & -4125 & $15 \cdot 496$ & $68 \cdot 7090$ \\
\hline
\end{tabular}

\section{Beidou G1 (\# 1) Beidou G3 (\# 3) Beidou G4 (\# 4)}
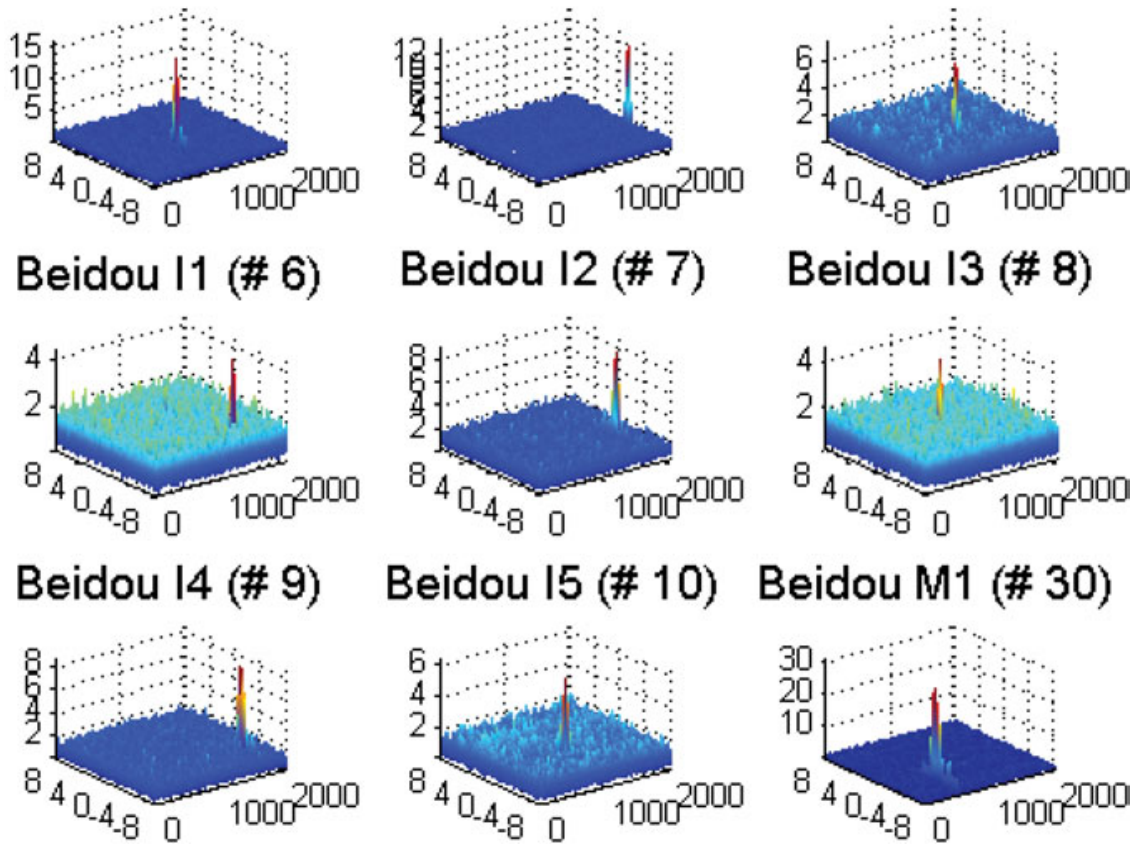

Figure 9. Acquisition diagram of Beidou satellite signals.

correlator output is plotted against the in-phase prompt correlator output while both are normalized by the mean magnitude of the prompt correlator output. Figure 11 also indicates that for a satellite signal that has a small $\mathrm{C} / \mathrm{N}$, the resulting signal is subject to more severe corruption. Nevertheless, all satellite signals can be successfully demodulated.

Since all satellite signals can be demodulated, the navigation data bits can then be accessed. By observing the results, some features of Beidou navigation signals and messages are observed. For Beidou GEO series satellites, the data rate appears to be $500 \mathrm{bps}$, implying that each bit duration is equivalent to twice the code period. This is in contrast to GPS L1 C/A signals in which the data 

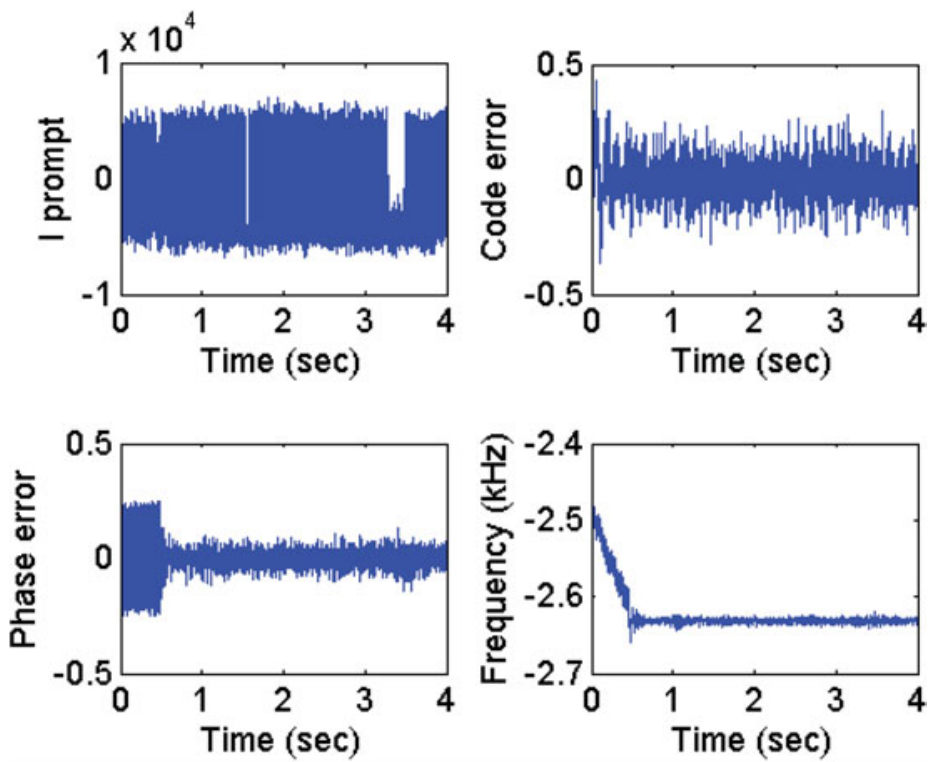

Figure 10. G1 signal tracking results.
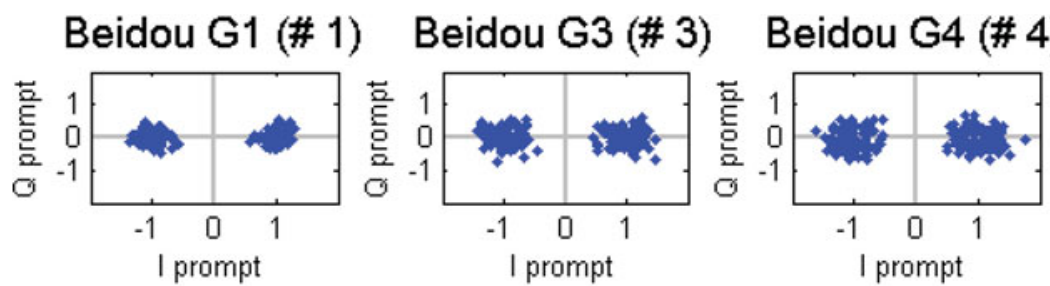

Beidou I1 (\# 6)

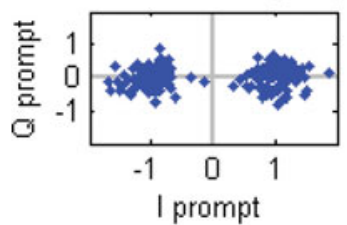

Beidou 12 (\# 7)

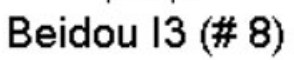

Beidou 14 (\# 9)
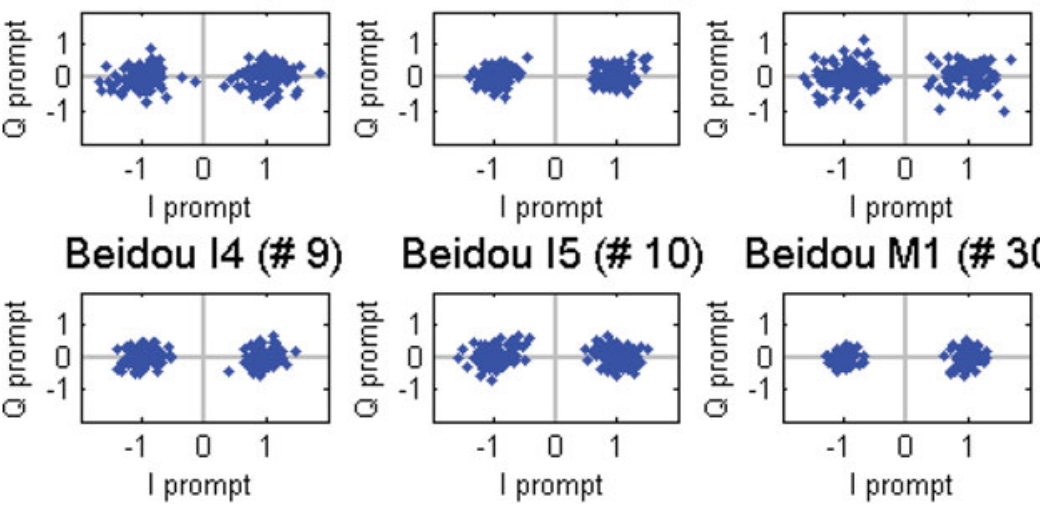

Figure 11. Beidou satellite signals signal constellation diagram.

rate is 50 bps while the code period is $1 \mathrm{msec}$. The use of higher data rate is believed to be advantageous in providing differential correction and integrity monitoring information. Since each data bit spans two ranging code periods, it is thus possible to 

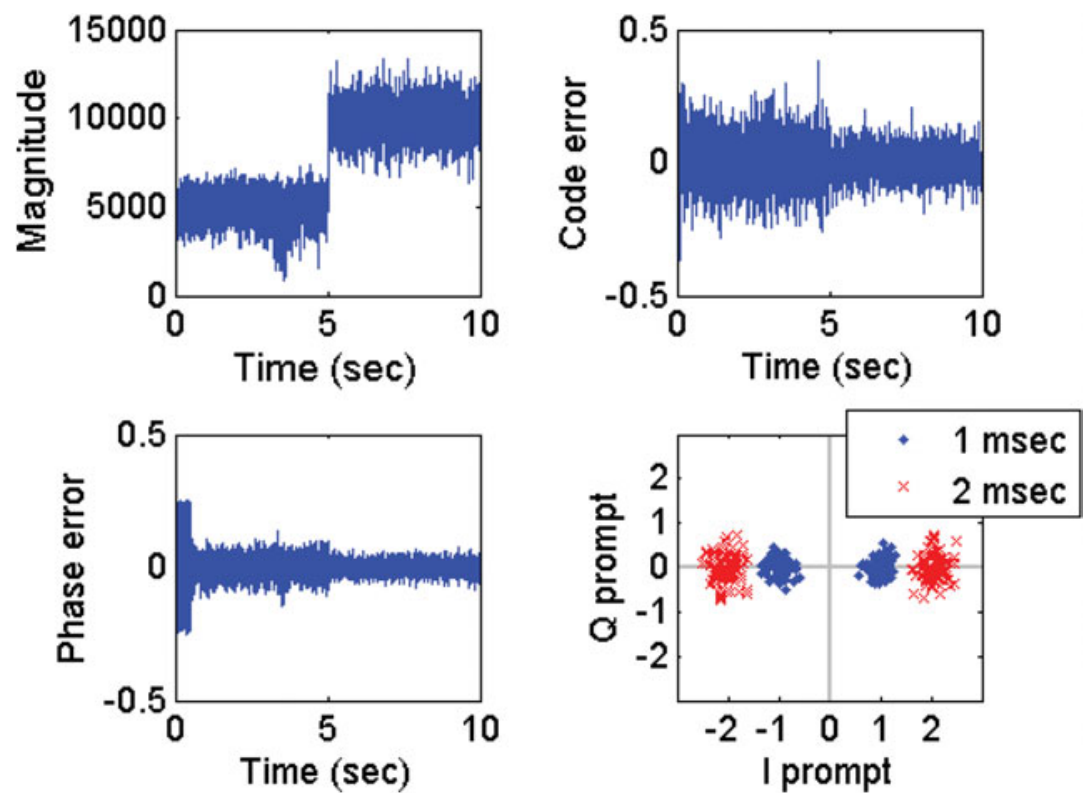

Figure 12. G1 satellite signal tracking.

increase the integration time to $2 \mathrm{msec}$ in the tracking operation. With the software receiver, such a modification can be easily made. Figure 12 depicts the constellation diagram with $1 \mathrm{msec}$ and $2 \mathrm{msec}$ integration times. In the figure, the switching from $1 \mathrm{msec}$ integration to $2 \mathrm{msec}$ integration is purposely set at $5 \mathrm{sec}$ for better illustration. Upon entering the lock state and passing the $5 \mathrm{sec}$ marker, the receiver matches the data bit pattern for the switch to a longer integration time. It is observed that the magnitude of the in-phase prompt correlator output is increased. The signal constellations before and after the switching are also illustrated. A better signal detection can be achieved by using the $2 \mathrm{msec}$ integration time.

By examining the tracking result of the Beidou MEO and IGSO series satellite signals with $1 \mathrm{msec}$ integration time, it is observed that the system employs secondary code to facilitate tracking and data decoding. This is the 20-bit NeumannHoffman code 00000100110101001110. As a result, the data rate of the MEO and GEO series satellite signals is 50 bps. In a similar manner, the software receiver is modified to take the secondary code into account to enhance tracking performance. Figure 13 depicts the magnitude of the in-phase prompt correlator output and the constellation diagram before (in which the integration time is $1 \mathrm{msec}$ ) and after (in which the integration time is $20 \mathrm{msec}$ ) the incorporation of the secondary code in signal tracking.

With the enhanced integration time, the raw navigation data messages can be reliably obtained. It is observed that each frame, as depicted in Figure 14, is subdivided into five 300-bit subframes. Each subframe begins with the telemetry word that includes the preamble. The remaining data messages are likely to contain clock, ephemeris, almanac, and other navigation related information. The data in Figure 14 were obtained in early 2012 before the release of the formal ICD (China Satellite 

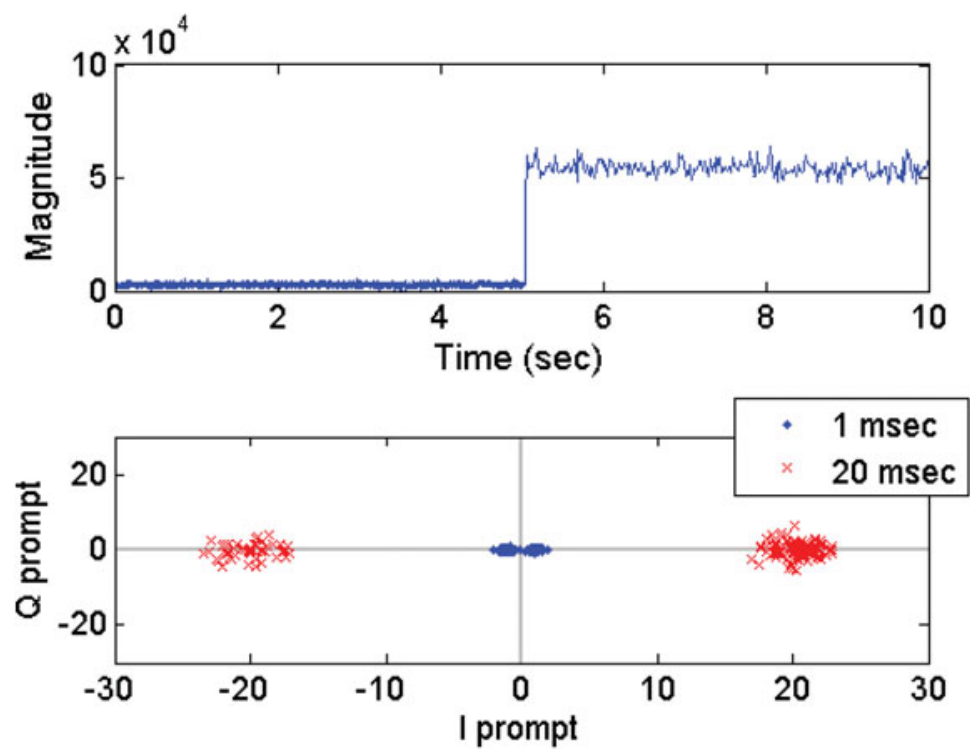

Figure 13. I1 satellite signal tracking.

300 bits per subframe

Subframe 1

\section{1}

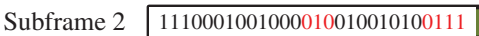

Subframe $3 \quad 1110001001000011010010101000$

Subframe $4 \longdiv { 1 1 1 0 0 0 1 0 0 1 0 0 0 1 0 0 0 1 0 0 1 0 1 0 0 0 1 1 }$

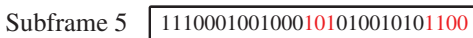

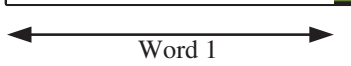

Figure 14. Arrangement of navigation data.

Navigation Office, 2012) in December 2012. The observed results are confirmed later to be the same as those described in the ICD.

4.2. Experiment 2. The software receiver was enhanced to perform data decoding and navigation tasks after the ICD was released. In the BDS, the arrangements of data messages of GEO and IGSO/MEO satellites are not identical. Nevertheless, based on the demodulated data, the subframes are aligned, the codes are decoded, the seconds-of-week are determined, and the ephemeris parameters are obtained. Accordingly, the positions of the satellites can be computed and, by using the pseudorange measurements, the user's position can be estimated. In this experiment, the antenna was fixed and the position estimates at 100 epochs with the interval of 1 second were computed. Four GEO and two IGSO satellites were used in position determination and the GDOP (geometric dilution of precision) is about $5 \cdot 31$. 


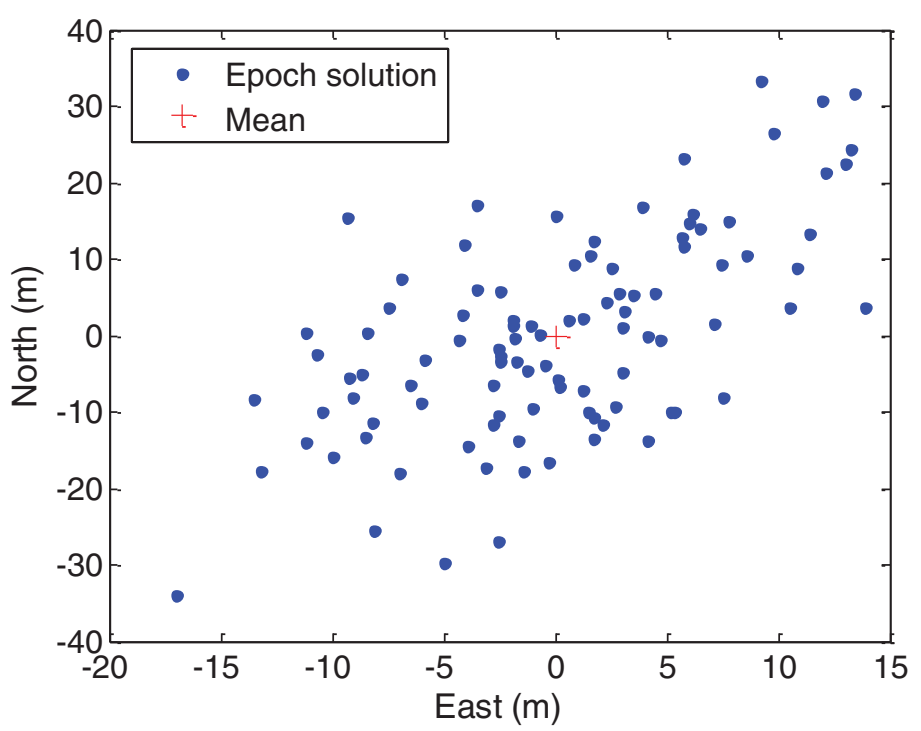

Figure 15. Horizontal error scatter plots.

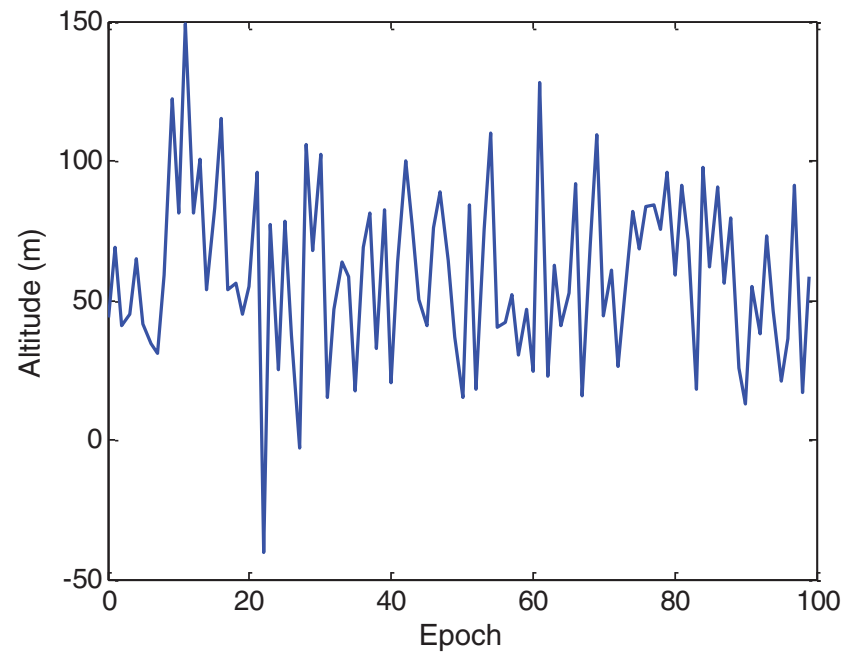

Figure 16. Altitude estimates.

The scatter plots in the horizontal plane are depicted in Figure 15 and the 2drms is about $29.97 \mathrm{~m}$. Figure 16 depicts the altitude estimates and the standard deviation is about $31.09 \mathrm{~m}$. The navigation function of the software receiver is thus verified.

5. CONCLUSION. This paper develops and applies software receiver techniques to receive Beidou navigation satellite signals. The flexibility of the software 
receiver enables the modification of receiver functions in the signal reception campaign to keep pace with the release of information. All Geostationary (GEO), Medium Earth Orbit (MEO), and Inclined Geostationary (IGSO) satellite signals are acquired and tracked by using the proposed software receiver. Differences between GEO and MEO/IGSO data arrangement are discussed. The positioning results of the PC-based software receiver are also reported and the Beidou navigation function is demonstrated. Given that we are entering the multi-Global Navigation Satellite System (GNSS) era, some satellite signal specifications may not be fully released and some may be subject to changes. The adopted software-defined radio (SDR) approach as demonstrated in the reception of Beidou signals enables a rapid assessment of the broadcast signals and can be valuable in providing insights to the signals for their better exploitation in multi-GNSS applications.

\section{ACKNOWLEDGEMENT}

The research was financially supported by National Science Council, Taiwan, under grant NSC 101-2221-E-006-191-MY3 and the National Cheug Kung University project of promoting academic excellence and developing world class research centres.

\section{REFERENCES}

Akos, D. M. (1997). A Software Radio Approach to Global Navigation Satellite System Receiver Design. Ph. D. Dissertation, Ohio University.

Beidou Navigation Satellite System. (2012). from http://www.beidou.gov.cn/

Borre, K., Akos, D. M., Bertelsen, N., Rinder, P., and Jensen, S. H. (2007). A Software-defined GPS and Galileo Receiver: A Single-Frequency Approach. Boston, MA, USA: Birkhaeuser.

Chen, Y. H., Juang, J. C., De Lorenzo, D. S., Seo, J., Lo, S., Enge, P., and Akos, D. M. (2011). Real-Time Dual-Frequency (L1/L5) GPS/WAAS Software Receiver. in The 24th International Technical Meeting of The Satellite Division of the Institute of Navigation (ION GNSS 2011).

China Satellite Navigation Office. (2011). Beidou Navigation Satellite System Signal in Space Interface Control Document (Test Version). Available at http://www.beidou.gov.cn/

China Satellite Navigation Office. (2012). Beidou Navigation Satellite System Signal in Space Interface Control Document: Open Service Signal B1I. Available at http://www.beidou.gov.cn/

Gao, G. X., Chen, A., Lo, S., De Lorenzo, D., Walter, T., and Enge, , P. (2009). Compass-M1 Broadcast Codes in E2, E5b, and E6 Frequency Bands. IEEE Journal of Selected Topics in Signal Processing, Vol. 3, pp. 599-612.

Hauschild, A., Montenbruck, O., Sleewaegen, J. M., Huisman, L., and Teunissen, P. (2012). Characterization of Compass M-1 Signals. GPS Solutions, Vol. 16, No. 1, pp. 117-126.

Heckler, G. W. and Garrison, J. L. (2006). SIMD Correlator Library for GNSS Software Receiver. GPS Solutions, Vol. 10, No. 4, pp. 269-276.

Holmes, J. K. (2007). Spread Spectrum Systems for GNSS and Wireless Communications. Boston: Artech House.

Juang, J. C. and Chen, Y. H. (2009a). Phase/Frequency Tracking in a GNSS Software Receiver. IEEE Journal of Selected Topics in Signal Processing, Vol. 4, No. 4, pp. 651-660.

Juang, J. C. and Chen, Y. H. (2009b). Accounting for Data Intermittency in a Software GNSS Receiver. IEEE Transactions on Consumer Electronics, Vol. 55, No. 2, pp. 327-333.

Juang, J. C. and Chen, Y.H. (2010). Global Navigation Satellite System Signal Acquisition Using Multi-bit Code and a Multi-layer Acquisition Strategy. IET Radar, Sonar, and Navigation, Vol. 4, No. 5, pp. 673-684.

Ledvina, B. M., Psiaki, M. L., Powell, S. P., and Kintner, P. (2004a). Bit-Wise Parallel Algorithms for Efficient Software Correlation Applied to a GPS Software Receiver. IEEE Transactions on Wireless Communications, Vol. 3, No. 5, pp. 1469-1473. 
Ledvina, B. M., Psiaki, M. L., Sheinfeld, D. J., Cerruti, A. P., Powell, S. P., and Kintner, P. M. (2004b). A Real-time GPS Civilian L1/L2 Software Receiver. in Proceedings of ION GNSS 2004, pp. 986-1005.

Sagiraju, P. K., Raju, G. S. V. and Akopian, D. (2008). Fast Acquisition Implementation for High Sensitivity Global Positioning Systems Receivers Based on Joint and Reduced Space Search. IET Radar, Sonar \& Navigation, Vol. 2, No. 5, pp. 376-387.

Tsui, J. (2000). Fundamentals of Global Positioning System Receivers: A Software Approach. New York: John-Wiley \& Sons. 